\title{
A RAPID SEMI-QUANTITATIVE DETERMINATION OF PHENYLALANINE, GLYCINE AND CYSTINE FROM URINE AND PHENYLALANINE FROM SERUM
}

\author{
Ming-liang LeE,* David A. ToKe, and Tso-Ren WANG** \\ Division of Medical Genetics, Department of Pediatrics, University of \\ Medicine \& Dentistry of New Jersey, Rutgers Medical School, \\ New Brunswick, New Jersey 08903 USA
}

\begin{abstract}
Summary Based on our previously described method of amino acid detection with fluorescamine, we described here a semi-quantitative determination of amino acid concentrations from urine and blood. The method is simple and rapid and is applicable for at least the urinary phenylalanine, glycine, cystine and the serum phenylalanine.
\end{abstract}

\section{INTRODUCTION}

Recently we described a micro technique for thin layer chromatography of amino acids from urine and serum using fluorescamine as a staining reagent (Wang et al., 1983). We report here the extension of this study to a rapid semi-quantitative determination of urinary and/or serum amino acid concentrations.

\section{MATERIALS AND METHODS}

All the material used is as described before (Wang et al., 1983). Chromatography and staining of amino acid were also as described (Wang et al., 1983).

Semi-quantitative estimation of amino acid concentration. Two methods were used depending on the availability of the sample. If more than $50 \mu 1$ of urine or serum is available, sample dilution method (method I) is more convenient. If not, reagent dilution method (method $\mathrm{II}$ ) is recommended.

Method I. Sample dilution method: Urine or serum was diluted in series (2, $4,6,8$ and 10 fold) with water. One tenth of microliter $(0.1 \mu l)$ from each dilution was chromatogramed in the same plate together with a series of known concentrations of amino acid. For example, for urinary or serum phenylalanine, $0.125,0.25$, 0.5 and $1.0 \mathrm{~mm}$ of phenylalanine were used. The lowest concentration of an amino

Received May 1, 1983

* To whom correspondence should be addressed.

** Present address: Department of Pediatrics, National Taiwan University, School of Medicine, Taipei, Taiwan. 
acid was chosen so that the fluorescamine staining would be negative (not visible or marginally visible). $0.25 \mathrm{~mm}$ phenylalanine usually yields positive fluorescence but $0.125 \mathrm{~mm}$ does not. Thus, the "end point" can be determined. By comparison of two end point concentrations, concentration of unknown sample can be estimated semiquantitatively.

Method II. Reagent dilution method: When sample available was not suffcient in quantity to do serial dilution, the following alternative was used. One tenth of a $\mu$ l of sample(s) was applied to 5 to 6 plates without dilution. In each plate, standard amino acid in a series of known concentrations were also applied. For example, for PKU serum, several plates were prepared. Each plate contained 0.1 fl of undiluted sample and phenylalanine in a concentration of $0.25,0.5,1.0,2.0$ and $3.0 \mathrm{~mm}$. The plates were then chromatographed in an ordinary fashion. After the chromatography each plate was stained with fluorescamine of different concentrations $(25,10,3.0,1.0,0.3$ and $0.1 \mathrm{mg} / 100 \mathrm{cc}$ acetone). By limiting the reagent concentration, an "end point" can be determined from the standard at each Fluram concentration. By comparing the end point of an unknown sample with those obtained from the known standard, the amino acid concentration in the unknown sample can be obtained (Table 1).

Table 1. Estimation of serum phenylalanine level by reagent dilution method. Whether the plates were read immediately after staining (A) or 30-60 min after (B), the phenylalanine concentration in unknown sample was estimated to be close to $2 \mathrm{~mm}(33 \mathrm{mg} \%)$. Plus, minus indicates presence or absence of fluorescent spot.

(A)

\begin{tabular}{|c|c|c|c|c|c|c|}
\hline \multirow{2}{*}{$\begin{array}{c}\text { Fluram } \\
\mathrm{mg}^{\circ} \%\end{array}$} & \multicolumn{5}{|c|}{ Phe, $\mathrm{mm}$} & \multirow{2}{*}{ Sample } \\
\hline & 3.0 & 2.0 & 1.0 & 0.5 & 0.25 & \\
\hline 25 & + & + & + & + & + & + \\
\hline 10 & + & + & + & $t$ & \pm & + \\
\hline 3.0 & + & + & + & - & - & + \\
\hline 1.0 & $\frac{1}{t}$ & + & - & - & - & \pm \\
\hline 0.3 & - & - & - & - & - & - \\
\hline 0.1 & - & - & - & - & - & - \\
\hline
\end{tabular}

(B)

\begin{tabular}{ccccccc} 
Fluram & \multicolumn{7}{c}{ Phe, mM } & Sample \\
\cline { 2 - 6 } $\mathrm{mg}^{0} \%$ & 3.0 & 2.0 & 1.0 & 0.5 & 0.25 & \\
\hline 25 & + & + & + & + & + & + \\
10 & + & + & + & + & + & + \\
3.0 & + & + & + & \pm & - & + \\
1.0 & + & + & \pm & - & - & \pm \\
0.3 & \pm & \pm & - & - & - & - \\
0.1 & \pm & - & - & - & - & \\
\hline
\end{tabular}

Jpn. J. Human Genet. 
Chromatography of radioactive amino acid. Five $\mu 1$ of undiluted radioactive $\left[{ }^{14} \mathrm{C}\right]$ phenylalanine (Wang et al., 1983) was added to $50 \mu \mathrm{l}$ of serum. One tenth of a microliter of this mixture was used for chromatography as described previously (Wang et al., 1983). Radioactivity was determined by (a) exposure of the plate to the ordinary Kodak film overnight and by (b) elution of radioactivity from the thin layer plate (cellulose of the thin layer plate was scraped in $0.5 \mathrm{~cm} /$ fraction and eluted with $0.5 \mathrm{cc}$ of water). Ten $\mu 1$ from each fraction was counted for recovery of the radioactivity.

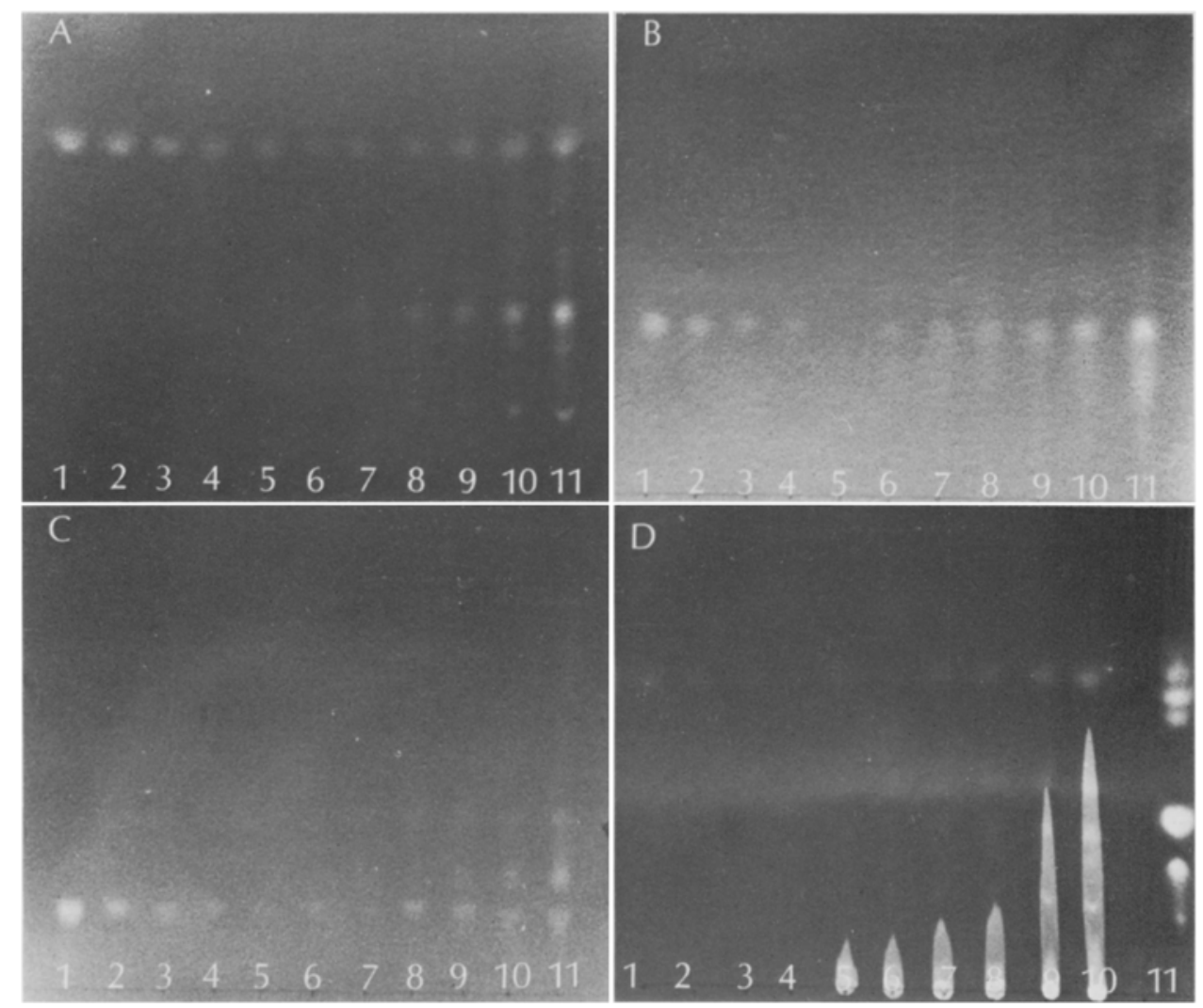

Fig. 1. Semi-quantitative estimation of amino acid concentrations by sample dilution method. (A) PKU urine: 1-6, phenylalanine 2.0, 1.5, 1.0, 0.75, 0.54, $0.25 \mathrm{~mm}$; $7-11$, urine diluted with water, 10, 8, 6, 4, 2 folds. (B) Hyperglycinuria: 1-4, glycine $1.0,0.75,0.5,0.25 \mathrm{~mm} ; 5-11$, urine diluted with water $16,14,12,10,8$, 6,4 folds. (C) Cystinuria: $1-5$, cystine $1.5,1.0,0.75,0.50,0.25 \mathrm{~mm} ; 6-11$ urine diluted with water $12,10,8,6,4,2$ folds. (D) PKU serum without deproteinization: $1-4$, phenylalanine $1.0,0.75,0.5,0.25 \mathrm{~mm} ; 5-9$, serum diluted with water 10 , $8,6,4,2$ folds; 10 , undiluted serum; 1 , standard amino acid mixture. Chromatographic procedures were as described in Wang et al. (1983). 


\section{RESULTS AND DISCUSSION}

Figure 1 shows the results by method I (sample dilution) applied to the estimation of concentration of urinary phenylalanine, glycine and cystine and serum phenylalanine. Table 1A and $1 \mathrm{~B}$ summarizes the results of concentration estimation by method II (reagent dilution method). Whether the plates were read immediately (Table $1 \mathrm{~A}$ ) or $30-60 \mathrm{~min}$ after the staining (Table $1 \mathrm{~B}$ ), the results were the same.

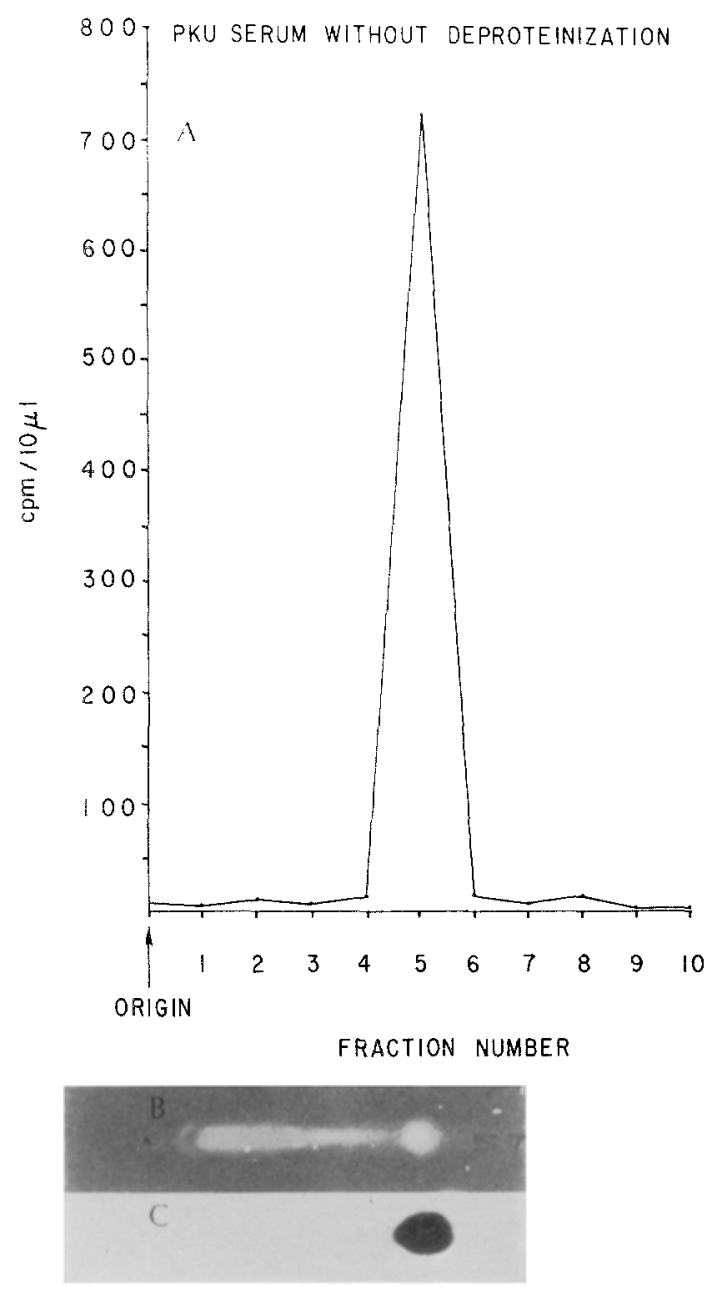

Fig. 2. Thin layer chromatography of undeproteinized PKU serum labeled with ${ }^{14} \mathrm{C}$-Phe. (A) Elution profile of radioactivity. (B) Fluorescamine staining. (C) Radioactivity as detected by exposing the plate to ordinary photographic film. For details, see METHOD. 
Since, ordinarily there is no difficulty in obtaining enough urine sample (greater than $50 \mu 1$ ), estimation of amino acid concentration by dilution method is recommended, as only one plate is needed. The application of the dilution method to serum may occasionally be difficult (when less than $50 \mu l$ of serum is available). In such circumstances, reagent dilution method (method II) can be used.

The estimated value by our method is approximately $\pm 20 \%$ of true value, a rough estimate yet acceptable in certain clinical situations. For example, this method can be used for management of PKU patients who come to the clinic for the follow-up of dietary restrictions. The phenylalanine level can be determined at the clinic before the patient sees the physician, thus proper dietary advice based on updated phenylalanine concentration can be made.

As described in our previous report (Wang et al., 1983), serum, if not deproteinized, gives a strong fluorescent streak. Therefore, amino acids which migrate in the streaked area could not be estimated. Although serum deproteinization (Wang et $a l$, 1983), can resolve the problem of streaking, minimum of $50 \mu 1$ of serum is needed. Furthermore, the estimation of amino acid concentrations required caution, since deproteinization results in 1.5-3-fold increase in amino acid concentration, depending on the individual amino acid (Wang et al., 1983). Addition of a known amount of radioactive amino acid before the deproteinization step to estimate the recovery of the procedure is an accurate way of following the concentration changes and can be used to correct the final estimate.

If non-deproteinized serum is used as in the case of phenylalanine, does the protein streak "trap" amino acid thus giving a falsely low estimation? In order to answer this question we co-chromatographed the radioactive amino acid with, for example, the PKU serum. As can be seen from Fig. 2, phenylalanine appears as a single fiuorescent (Fig. 2B) and single radioactive spot (Fig. 2C). There was no radioactive streaking. The recovery of radioactivity from a single fraction (fraction 5) spot was $93 \%$. Therefore, the effect of serum protein in the migration of phenylalanine, is minimum. This lack of interference from serum protein will most likely be true for those amino acids migrating further than the protein streak (Leu, Ile, Val, Try, Met, Tyr). Therefore, our method can be potentially used for undeproteinized serum for the above amino acids.

Acknowledgement The authors would like to thank Dr. C.Y. Lai for his reading and criticism of the manuscript.

\section{REFERENCE}

Wang, T.R., Lee, M.L., and Toke, D. 1983. A micro thin layer chromatography technique for urinary and serum amino acids using fluorescamine as a staining reagent. Jpn. J. Human Genet. 28: 273-284. 